\title{
ASYMPTOTIC EQUALITY OF THE ISOLATED AND THE ADIABATIC SUSCEPTIBILITY
}

\author{
T.P. VALKERING and W.J. CASPERS
}

Afdeling der Technische Natuurkunde van de Technische Hogeschool Twente, Enschede, The Netherlands

Received 26 August 1974

\section{Synopsis}

Many-particle systems with a hamiltonian of the form $H=A+h B, h$ being a parameter, are discussed. In particular, for a certain class of these systems, a criterion is derived for the asymptotic equality of the isolated and the adiabatic susceptibility or, equivalently, for the ergodicity of $B$. This criterion states that, for sufficiently large particle number, any hermitian operator polynomial in $h$ of any degree $J$ that commutes with $H(h)$ can be written as a linear combination of the powers $H^{0}, \ldots, H^{J}$ with polynomial coefficients.

1. Introduction. This paper deals with many-particle systems with a hamiltonian $H$ that depends on a real parameter $h$. To describe the rate of change of the canonical average $\langle\mathrm{d} H / \mathrm{d} h\rangle$ for an isolated process, two susceptibilities were introduced in the literature ${ }^{1,2}$ ): the so-called isolated and the adiabatic susceptibility. Generally the difference between these susceptibilities does not equal zero. Even in the many-particle limit this difference is not negligibly small for all hamiltonians ${ }^{1-3}$ ).

In a series of papers conditions have been discussed that are sufficient for the asymptotic equality of both susceptibilities. In particular we mention results of Klein $^{4}$ ) and Caspers ${ }^{5}$ ), who found conditions in terms of properties of the eigenvalues of $H(h)$. In the present paper a sufficient condition is derived, which is intimately related to these results. This relation will be discussed in section 5 .

Broer $^{2}$ ) and Yamamoto ${ }^{6}$ ) found ergodicity of $\mathrm{d} H / \mathrm{d} h$ to be a sufficient condition. In fact ergodicity of $\mathrm{d} H / \mathrm{d} h$, as is formulated by Mazur $^{7}$ ), is equivalent to the asymptotic equality of the susceptibilities.

In this paper we restrict ourselves to systems with a hamiltonian of the form

$$
H(h)=A+h B \text {. }
$$

The operators $A$ and $h B$ are hermitian and are defined on a Hilbert space of finite dimension $n$. They represent respectively the interaction between the particles of 
the system and the energy of these particles in some external field. The strength of the field is proportional to $h$. The canonical average of $B$ is supposed to be an extensive quantity. An array of $N$ similar spins in a magnetic field is a typical example of such a system.

Any operator of the type (1.1) has a spectral decomposition ${ }^{8}$ )

$$
H(h)=\sum_{i=1}^{m} \varepsilon_{i}(h) P_{i}(h)
$$

The $m$ symbols $\varepsilon_{l}(h)$ denote different real analytic functions of $h$, which represent the eigenvalues of $H(h)$. The $P_{i}(h)$ denote the corresponding eigenprojections, which are analytic vector-valued functions. We suppose in this analysis that $m=n$. In that case each $P_{i}(h)$ projects on a one-dimensional subspace. In section 5 it will be argued that this restriction is not essential.

Now we shall formulate what is meant by asymptotic equality of the isolated and the adiabatic susceptibility. The canonical density operator has a spectral decomposition

$$
\varrho(T, h)=\sum_{i=1}^{n} \varrho_{i}(T, h) P_{i}(h)
$$

where $T$ denotes the temperature. The isolated and the adiabatic susceptibilities are respectively defined ${ }^{2}$ ) by

$$
\begin{aligned}
& \chi_{\text {iso }}=-\sum_{i=1}^{n} \varrho_{i}\left(\frac{\mathrm{d}}{\mathrm{d} h}\right)^{2} \varepsilon_{i}, \\
& \chi_{\mathrm{s}}=-\left(\frac{\partial}{\partial h} \sum_{i=1}^{n} \varrho_{i} \frac{\mathrm{d}}{\mathrm{d} h} \varepsilon_{i}\right)_{s}=\left(\frac{\partial}{\partial h}\langle-B\rangle\right)_{\mathrm{s}},
\end{aligned}
$$

where $S$ denotes the entropy. The susceptibilities being extensive quantities, one can say that they are asymptotically equal if

$$
\lim _{N \rightarrow \infty} N^{-1}\left(\chi_{\text {sN }}-\chi_{\text {iso } N}\right)=0
$$

The hamiltonian and the quantities derived from it depend parametrically on the particle number. If necessary this will be indicated with the suffix $N$.

The argument in this paper runs as follows. In section 2 we give some results from papers by $\mathrm{Mazur}^{7}$ ) and by Caspers et $a .^{9}{ }^{9}$ ), which are necessary for our analysis. It is of particular importance that $\chi_{\mathrm{s}}-\chi_{\text {iso }}$ can be written as the square of the norm of an element of the Hilbert space introduced in section 2 . In section 3 a particular set of operator polynomials of the form $\sum_{i=0}^{k} G_{i} h^{l}$ that commute 
with $H(h)$ is discussed. The results of this section are taken partly from the refs. 10 and 11. In section 4 this set of polynomials is used to construct an orthogonal decomposition of the above-mentioned Hilbert space, so that $\chi_{s}-\chi_{\text {iso }}$ can be written as a sum of non-negative terms. This allows the formulation of a condition that is sufficient for the asymptotic equality of $\chi_{\mathrm{s}}$ and $\chi_{\text {iso }}$ for a certain class of systems. In section 5 a discussion is given of the class mentioned, of the restriction to hamiltonians with $n$ different energy levels $\varepsilon_{i}(h)$, and of the relation between the criterion and level crossing.

2. An expression for $\chi_{\mathrm{s}}-\chi_{\mathrm{iso}}$. In this section $h$ has an arbitrary but fixed value. The hermitian operators that commute with $H$, for a particular $N$, constitute a linear space over the real numbers. An inner product $(D, C)$ of two elements $D$ and $C$ of this space can be defined as the canonical average of their product

$$
(D, C)=\langle D C\rangle
$$

With this inner product this space is a Hilbert space, which will be denoted by $\mathscr{C}^{\neq}$.

Define the following operators in $\mathscr{C}$

$$
Q_{0}=I, \quad Q_{1}=(H-\langle H\rangle I) /\left(\left\langle H^{2}\right\rangle-\langle H\rangle^{2}\right)^{\frac{1}{2}},
$$

where $I$ denotes the indentity, and

$$
\begin{aligned}
B_{C} & =\sum_{i=1}^{n}\left[\frac{\mathrm{d}}{\mathrm{d} h} \varepsilon_{i}(h)\right] P_{i}(h), \\
C_{N} & =N^{-\frac{1}{2}}\left(B_{C N}-\left\langle B_{C N} Q_{0 N}\right\rangle Q_{0 N}-\left\langle B_{C N} Q_{1 N}\right\rangle Q_{1 N}\right) .
\end{aligned}
$$

Then holds

$$
N^{-1}\left(\chi_{\mathrm{SN}}-\chi_{\mathrm{iso} N}\right)=\left\langle C_{N}^{2}\right\rangle
$$

which identity was derived by Broer ${ }^{2}$ ). Obviously $C_{N}$ is an element of $\mathscr{C}_{N}$, thus $N^{-1}\left(\chi_{\mathrm{SN}}-\chi_{\text {iso } N}\right)$ equals the square of the norm of a vector in this space.

Mazur ${ }^{7}$ ) derived $\lim _{N \rightarrow \infty}\left\langle C_{N}^{2}\right\rangle=0$ as a criterion for ergodicity of $\mathrm{d} H / \mathrm{d} h$. In particular he showed for the one-dimensional $X-Y$ model that the length of the projection of $C_{N}$ on a certain subspace of $\mathscr{C}_{N}$ does not tend to zero in the manyparticle limit, so that the magnetization is nonergodic in this model. For a detailed discussion of $\lim _{N \rightarrow \infty}\left\langle C_{N}^{2}\right\rangle=0$ as a criterion for ergodicity, we refer to ref. 11 .

Now we shall give an important result of ref. 9. Consider the set $\left\{H^{0}, H^{1}, \ldots, H^{l}\right\}$ where $l$ is the largest integer for which this set is linearly independent. For almost

₹ An interesting extension to the case of operators defined in a separable infinite-dimensional Hilbert space has been given in ref. 12 . 
all $h, l$ equals $n-1$. Only for values of $h$ for which $H$ has less than $n$ different eigenvalues, $l$ is smaller. With this set of powers of $H$ an orthonormal set in $\mathscr{C}$ can be constructed

$$
\begin{gathered}
Q_{0}=H^{0}, \quad Q_{i}=\left(H^{i}-\sum_{j=0}^{i-1}\left\langle Q_{j} H^{i}\right\rangle Q_{j}\right) /\left\langle\left(H^{i}-\sum_{j=0}^{i-1}\left\langle Q_{j} H^{i}\right\rangle Q_{j}\right)^{2}\right\rangle^{\frac{1}{2}} \\
i=1, \ldots, l
\end{gathered}
$$

If $l$ equals $n-1$ the set $\left\{Q_{i}\right\}$ is an orthonormal basis in $\mathscr{C}$. Starting from the following asymptotic order of magnitude relations

$$
\left[\left(\frac{\partial}{\partial T}\right)^{i}\left\langle H_{N}\right\rangle\right]_{h}=\mathcal{O}(N), \quad\left[\left(\frac{\partial}{\partial T}\right)^{i}\left\langle B_{N}\right\rangle\right]_{h}=\mathcal{O}(N),
$$

Caspers et al. ${ }^{9}$ ) proved

$$
\left\langle C_{N} Q_{i N}\right\rangle^{2}=\mathcal{O}\left(N^{-i+1}\right), \quad i=2,3, \ldots
$$

These relations are the basis for the analysis in section 4.

3. The minimal basis. Consider the hermitian operators $G(h)$ of the form

$$
G(h)=a(h) \sum_{i=0}^{k} G_{i} h^{l}
$$

that commute with $H(h)$. The $G_{l}$ denote constant hermitian operators, $a(h)$ is an element of the field $\mathbf{R}(h)$ of rational expressions in $h$ with real coefficients and $k$ is an arbitrary non-negative integer. These operators $G(h)$ constitute a linear space over $\mathbf{R}(h)$, which will be called $\mathscr{K}$. In contrast with the foregoing section $h$ is considered here as a variable. It is not difficult to see that, on account of the assumption $m=n$ in the spectral decomposition (1.2) of $H(h)$, the dimension of $\mathscr{K}$ equals $n$ and that the powers $H^{0}, \ldots, H^{n-1}$ form a basis. Any set of $n$ linearly independent polynomials is a basis. This section is devoted to properties of a particular class of polynomial bases, which are defined as follows.

Definition 3-1. The sum of the degrees of the elements of a polynomial basis in $\mathscr{K}$ is called the degree of the basis. A polynomial basis is called minimal if there is no basis with lower degree.

Minimal bases were discussed extensively in the refs. 10 and 11. The following three theorems are identical with or immediate consequences of respectively theorem $2-3$, the corollary thereafter and theorem $2-4$ of ref. 10 . Here they are given without proof. 
Theorem 3-2. The degree of a minimal basis equals $\frac{1}{2} n(n-1)-q, q$ being the number of linearly independent linear relations between the symmetrized products of $A$ and $B$ of order $0, \ldots, n-1$.

The different symmetrized products of $A$ and $B$ of order $k,\left\{A^{k-j} B^{j}\right\}$ with $j=0, \ldots, k$, are defined as the coefficients of the corresponding $j$ th power of $h$ in $(A+h B)^{k}$.

Theorem 3-3. A polynomial basis with elements

$$
L_{i}(h)=\sum_{j=1}^{m_{i}} G_{i j} h^{j}, \quad i=1, \ldots, n,
$$

is minimal if and only if the set of hermitian operators $\left\{G_{i j} \mid i=1, \ldots, n\right.$; $\left.j=0, \ldots, m_{l}\right\}$ is linearly independent.

Theorem 3-4. Let $\left\{L_{i}^{\prime}\right\}$ and $\left\{L_{i}^{\prime \prime}\right\}$ be minimal bases. If the basis elements are ordered according to increasing degree it follows that

$$
\text { degree } L_{i}^{\prime}=\text { degree } L_{i}^{\prime \prime} \text {. }
$$

The following theorem shows the particular character of a minimal basis as compared with the other bases.

Theorem 3-5. Let $\left\{L_{i}(h)\right\}$ be a minimal basis with individual degrees $m_{i}$. Then any hermitian matrix polynomial $P(h)$ with degree, say, $s$ in $\mathscr{K}$ can be written as

$$
P(h)=\sum_{i \in \mathbf{S}} \gamma_{i}(h) L_{i}(h),
$$

where the coefficients $\gamma_{i}(h)$ are real polynomials and $S$ denotes the index set $\left\{i \mid m_{i} \leq s\right\}$. Further it follows that

$$
m_{i}+\text { degree } \gamma_{i}(h) \leq s, \quad i \in S .
$$

Proof. The set $\left\{L_{i}(h)\right\}$ being a basis in $\mathscr{K}$, the polynomial $P(h)$ surely can be written as

$$
P(h)=\sum_{i=1}^{n} \gamma_{i}(h) L_{i}(h), \quad \gamma_{i}(h) \in \mathbf{R}(h)
$$

The coefficients $\gamma_{i}(h)$ are polynomials as we shall show now.

Let $\psi(h)$ be the least common multiple of the denominators of the $\gamma_{i}(h)$. Multiplying (3.6) by $\psi(h)$ we get

$$
\psi(h) P(h)=\sum_{i=1}^{n} \psi(h) \gamma_{i}(h) L_{l}(h)
$$


Let $\psi(h)$ have a zero for some $h=h^{\prime}$. Surely not all polynomials $\psi(h) \gamma_{i}(h)$ have a zero for this $h^{\prime}$. Substitution of $h^{\prime}$ in (3.7) then leads to a nontrivial linear relation between the operators $G_{i j}$. This is in contradiction ( $c f$. theorem 3-3) with the assumption that the set $\left\{L_{i}(h)\right\}$ is a minimal basis. So we conclude that $\psi(h)$ has no zeros and so the coefficients $\gamma_{t}(h)$ are polynomials.

Assuming that either (3.5) is not true or that there is a nonzero coefficient $\gamma_{l}(h)$ with $m_{i}>s$, one also finds a nontrivial linear relation in the set $\left\{G_{i j}\right\}$. This completes the proof of the theorem.

The last theorem of this section is necessary for the proof of the main result of this paper, formulated in theorem 4-1.

Theorem 3-6. Suppose that there is a non-negative integer $j \leq n-1$ so that each polynomial operator $P(h)$ in $\mathscr{K}$ with degree $s \leq j$ can be written as

$$
P(h)=\sum_{i=0}^{s} \gamma_{i}(h) H^{t}(h)
$$

where the coefficients $\gamma_{i}(h)$ are polynomials. If this condition is satisfied there is a minimal basis $\left\{H^{0}, H, \ldots, H^{j}, L_{j+2}, \ldots, L_{n}\right\}$, with the property that degree $L_{i}>j$ for $i=j+2, \ldots, n$.

Proof. Let $\left\{H^{0}, L_{2}, \ldots, L_{n}\right\}$ be a minimal basis, ordered according to increasing degree. Obviously such a minimal basis exists. Now suppose that the degree of $L_{2}$ equals zero. As a consequence of the assumption (3.8) $L_{2}=\gamma_{0} H^{0}$ then holds. This is in contradiction with the fact that both $L_{2}$ and $H^{0}$ are elements of a basis in $\mathscr{K}$, so that degree $L_{2} \geq 1$. Because we have assumed that degree $L_{i}$ $\leq$ degree $L_{i+1}$, this completes the proof of the theorem if $j=0$.

If $j \geq 1$ we proceed in the following way. The assumption that the degree of $L_{2}$ is larger than unity leads, with theorem 3-5, to the relation $H=\gamma_{0} H^{0}$. This result, however, is in contradiction with our assumption that $H$ has $n$ different energy levels $\varepsilon_{i}(h)$ [cf. (1.2) and following]. So we conclude that the degree of $L_{2}$ equals 1 . On account of (3.8) we can write

$$
L_{2}=\gamma_{0} \dot{H}^{0}+\gamma_{1} H^{1}
$$

The coefficient $\gamma_{1}$ is surely unequal to zero. Consequently $\left\{H^{0}, H^{1}, L_{3}, \ldots, L_{n}\right\}$ is a basis in $\mathscr{K}$, which is minimal because it has the same degree as the minimal basis $\left\{H^{0}, L_{2}, \ldots, L_{n}\right\}$. The proof of the inequality degree $L_{3}>1$ is analogous to that of degree $L_{2}>0$, given above. This completes the proof of the theorem if $j=1$. If $j \geq 2$ we proceed in an analogous way. 
4. Sufficient conditions for asymptotic equality. With the aid of a minimal basis an orthogonal decomposition of the Hilbert space $\mathscr{C}$, defined in section 2 , can be constructed. In terms of this decomposition $\left\langle C_{N}^{2}\right\rangle[c f .(2.3)$ and (2.4)] can be written as a sum of non-negative terms.

Let $\left\{L_{i}\right\}$ be a minimal basis, ordered according to increasing degree. For any particular value of $h$ the operators $L_{i}$ are elements of $\mathscr{C}$. Define the subspaces $\mathscr{V}_{i}$ as follows

$$
\mathscr{V}_{i}=\operatorname{span}\left\{L_{j} \mid \text { degree } L_{j}=i\right\}, \quad i=0,1, \ldots
$$

Orthogonal subspaces of $\mathscr{C}$ can then be defined

$$
\mathscr{W}_{0}=\mathscr{V}_{0}, \quad \mathscr{W}_{i}=\operatorname{span}\left\{G-\sum_{j=0}^{i-1} \hat{P}_{j} G \mid G \in \mathscr{V}_{i}\right\}, \quad i=1,2, \ldots,
$$

where $\widehat{P}_{i}$ denotes the orthogonal projection on $\mathscr{W}_{i}$. Making use of the uniqueness theorem 3-4 one can easily see that the subspaces $\mathscr{W}_{i}$ are unique, although the spaces $\mathscr{V}_{i}$ depend on the particular choice of the minimal basis. With the exception of values of $h$ for which $H$ has less than $n$ different eigenvalues, the spaces $\left\{\mathscr{W}_{i}\right\}$ span $\mathscr{C}$.

The set $\left\{L_{i}\right\}$ being a minimal basis one can show, however, that for any $h$ the operator $C$, defined in (2.3), is a linear combination of the $L_{i}$. It follows from (2.4) that

$$
N^{-1}\left(\chi_{\mathrm{sN}}-\chi_{\mathrm{iso} N}\right)=\sum_{i=0}^{\infty}\left\langle\left(\hat{P}_{i N} C_{N}\right)^{2}\right\rangle
$$

We conclude from this relation that it is necessary and sufficient for (1.6) to be true that

$$
\left.\lim _{N \rightarrow \infty}\left\langle\hat{P}_{i N} C_{N}\right)^{2}\right\rangle=0, \quad i=0,1, \ldots
$$

and that for any $\varepsilon>0$ there is an integer $J$ such that for all $N$

$$
\sum_{j+1}^{\infty}\left\langle\left(\hat{P}_{i N} C_{N}\right)^{2}\right\rangle<\varepsilon
$$

If we ask for sufficient conditions only, the equality (4.4) can be replaced by a condition in terms of properties of the hamiltonian and its commuting polynomials. We shall formulate this condition for a particular class of hamiltonians. This class is called $\Gamma$ and contains precisely those hamiltonians for which (4.5) holds for all the values of $h$ and $T$ for which the asymptotic order relations (2.6) are valid. 
The orem 4-1. Let $h$ and $T$ have such a value that the relations (2.6) hold and let the hamiltonian be in $\Gamma$.

Then $\lim _{N \rightarrow \infty} N^{-1}\left(\chi_{\mathrm{s} N}-\chi_{\text {iso } N}\right)$ holds if for any integer $s$ there is an integer $N_{s}$ such that for $N>N_{s}$ each polynomial $P(h)$ of degree $s$ that commutes with $H_{N}(h)$ can be written as

$$
P(h)=\sum_{i=0}^{s} \gamma_{i}(h) H_{N}^{i}(h),
$$

with polynomial coefficients $\gamma_{i}(h)$.

Note. In (4.6) $h$ has to be considered as a variable as in section 3 .

Proof. We shall show that (2.6) and (4.6) together imply (4.4). This proves the theorem. For any $s$ there is a number $N_{s}$ such that (4.6) is valid. Define for any $J$ the number $N_{J}^{\prime}=\max \left(N_{0}, \ldots, N_{J}\right)$. Any polynomial $P(h)$ of degree $s=0, \ldots, J$ that commutes with $H_{N}$ can then be written as in (4.6) for $N>N_{J}^{\prime}$. Theorem 3-6 can then be applied and we conclude that for any $J$ there is an integer $N_{J}^{\prime}$ such that for all $N>N_{J}^{\prime}$ there is a minimal basis $\left\{H^{\circ}, \ldots, H_{J}, L_{J+2}, \ldots, L_{n}\right\}$. Because it also holds that the degree of $L_{i}$ is larger than $J$ for $i \geq J+2$ the spaces $\mathscr{W}_{0}, \ldots, \mathscr{W}_{J}$ are spanned respectively by the vectors $Q_{0}, \ldots, Q_{J}$, defined in (2.5). As a consequence $\left\langle\left(\widehat{P}_{i} C\right)^{2}\right\rangle=\left\langle Q_{i} C\right\rangle^{2}$ holds for $i=0,1, \ldots, J$ and $N>N_{J}^{\prime}$. With $\left\langle Q_{0} C\right\rangle=\left\langle Q_{1} C\right\rangle=0$ and with (2.6) follows (2.7), so that (4.4) holds.

It is relevant to remark that for arbitrarily chosen $A$ and $B$ there are in general no nontrivial linear relations between the symmetrized products of $A$ and $B$ of order $0, \ldots, n-1^{13}$ ). In that case $q$ in theorem 3-2 equals zero so that the set $H^{0}, \ldots, H^{n-1}$ is a minimal basis. In fact one can even show ${ }^{11}$ ) that the criterion in theorem 4-1 is satisfied if there is a number $\alpha<1$ so that $q_{N} \leq \alpha n_{N}$ holds for sufficiently large $N$. This supports the point of view that (1.6) holds for realistic hamiltonians.

5. Discussion. The criterion derived for the asymptotic equality of $\chi_{\mathrm{s}}$ and $\chi_{\text {iso }}$, is only applicable for a restricted class of systems. The hamiltonian has to be in $\Gamma$ and must have $n$ different eigenvalues $\varepsilon_{i}(h)$ for almost all $h$. Discussion of these restrictions and of the relation of the criterion with the nonoccurrence of level crossing, form the contents of this section.

Theorem 4-1 is only of interest if $\Gamma$ can be identified with a large class of physically interesting hamiltonians. To begin with, it surely contains the hamiltonians for which (1.6) is satisfied, because (4.5), which defines $\Gamma$, is a necessary condition for (1.6). Theorem 4-1 would be trivial, however, if $\Gamma$ would contain no other hamiltonians of interest. Indeed it contains other hamiltonians as we shall show now. 
Consider the hamiltonian of a system consisting of two non-interacting subsystems in the same external field

$$
H=H_{1} \otimes I_{2}+I_{1} \otimes H_{2},
$$

and suppose that (1.6) holds separately for each hamiltonian. It is not difficult to prove ( $c f$. ref. 9) that generally (1.6) does not hold for the combined system. The hamiltonian $H$ in (5.1), however, belongs to $\Gamma$ as will be shown now.

From the relations $C=C_{1} \otimes I_{2}+I_{1} \otimes C_{2}$ and $\left\langle C_{1} C_{2}\right\rangle=\left\langle C_{1}\right\rangle\left\langle C_{2}\right\rangle=0$ follows

$$
\left\langle C^{2}\right\rangle=\left\langle C_{1}^{2}\right\rangle+\left\langle C_{2}^{2}\right\rangle
$$

By assumption there is a $J$ for any positive $\varepsilon$ so that for all $N_{1,2}$

$$
\left.\sum_{j+1}^{\infty}\left\langle\hat{P}_{i s} C_{s}\right)^{2}\right\rangle<\varepsilon, \quad s=1,2 .
$$

If $\left\{L_{i 1,2}\right\}$ are minimal bases for the separate systems, a minimal basis for the combined system is the set $\left\{L_{i 1} \otimes L_{j 2} \mid i=1, \ldots, n_{1} ; j=1, \ldots, n_{2}\right\}^{11}$ ). Eq. (5.3) means that $C_{1} \otimes I_{2}$ and $I_{1} \otimes C_{2}$ are approximated by. respectively the basis elements $\left\{L_{i 1} \otimes I_{2} \mid i=1, \ldots, J\right\}$ and $\left\{I_{1} \otimes L_{i 2} \mid i=1, \ldots, J\right\}$. We conclude on account of (5.2) that for all $N$

$$
\sum_{J+1}^{\infty}\left\langle\left(\widehat{P}_{i} C\right)^{2}\right\rangle<2 \varepsilon
$$

so that the combined hamiltonian belongs to $\Gamma$.

An analogous situation arises if there is a constant operator that commutes with $H(h)$. Then the Hilbert space splits up into two invariant subspaces and the hamiltonian is the direct sum of its restrictions to these subspaces. An argument analogous to the foregoing one can be given.

Thus for these well-known examples of nonergodic behaviour, the property (1.6) breaks down for the total hamiltonian, whereas (4.5) remains valid. This leads to the hypothesis that (4.5) can be proved for the larger class of many-particle systems that are of interest in statistical mechanics. So far, however, we were not able to prove this hypothesis. A more detailed discussion of this problem can be found in ref. 11.

The restriction to hamiltonians with $n$ different eigenvalues $\varepsilon_{i}(h)$ is used in fact only in section 3 . We shall outline now the basis of a formalism in which this restriction does not appear. Results analogous to those given in section 3 can be proved by straight-forward generalization of the argument.

Suppose there are $m$ nonidentical functions $\left\{\varepsilon_{i}(h) \mid i=1, \ldots, m\right\}$ that represent the eigenvalues of $\boldsymbol{H}(h)$. Then the operators $\left\{H^{i-1} \mid i=1, \ldots, m\right\}$ are linearly 
independent over the field of rational expressions in $h$ with real coefficients, $\mathbf{R}(h)$. These powers then span a subspace of $\mathscr{K}$. The space $\mathscr{K}$ has been defined in section 3 . This subspace, denoted by $\mathscr{K}_{s}$, has a dimension $m$. In the same way as in $\mathscr{K}$ (definition 3-1) a minimal basis can be defined in $\mathscr{K}_{s}$. With a minimal basis orthonormal subspaces $\mathscr{W}_{i}$ can be defined and so we arrive at the relation(4.3). In the argument $n$ has to be replaced by $m$ and the polynomial $P(h)$ occurring in the theorems 3-5, 3-6 and 4-1 has to be chosen from $\mathscr{K}_{s}$. So we may conclude that the restriction discussed here does not have severe consequences.

This last section is concluded with a discussion of the relation of the criterion in theorem 4-1 with crossing of the energy levels $\varepsilon_{i}(h)$. As is indicated in the proof of this theorem, the following relations hold for the degrees of the elements of a minimal basis, if the criterion is satisfied,

$$
\begin{aligned}
& \text { degree } L_{l}=i-1, \quad i=1, \ldots, J+1, \\
& \text { degree } L_{l} \geq J+1, \quad i=J+2, \ldots, n .
\end{aligned}
$$

The degree of the minimal basis being equal to $\frac{1}{2} n(n-1)-q(c f$. theorem 3-2), it follows that $\frac{1}{2} n(n-1)-q \geq 0+1+2+\cdots+J+(J+1)(n-J-1)$, or

$$
q \leq \frac{1}{2}(n-J-2)(n-J-1) .
$$

The number of level crossings does not exceed $q(c f$. refs. $10,11,13)$ so that the criterion implies an upper bound for this number.

This result is in agreement with the heuristic conclusion which can be drawn from ref. 5, that (1.6) holds if the number of level crossings does not exceed a certain number, which is unknown however. This conclusion is based on the hypothesis II in this reference, which states that there exists a continuous function $g(\alpha, h)$ so that for sufficiently large $N$ the slopes $N^{-\frac{1}{2}} \mathrm{~d} \varepsilon_{i N} / \mathrm{d} h$ are approximated by $g\left(N^{-\frac{1}{2}} \varepsilon_{t N}, h\right)$. The argument runs as follows: the level density increases with $N$. If the levels should not cross, the slopes of neighbouring levels will tend to be equal for large $N$ so that such a function $g(\alpha, h)$ indeed will exist. However, a certain number of crossings may be admitted because $g(\alpha, h)$ only approximates the derivatives $\mathrm{d} \varepsilon_{t} / \mathrm{d} h$.

\section{REFERENCES}

1) Wright, A., Phys. Rev. 76 (1949) 1826.

2) Broer, L.J. F., Physica 17 (1951) 531.

3) Caspers, W.J., Physica 25 (1959) 43.

4) Klein, M.J., Phys. Rev. 86 (1952) 807.

5) Caspers, W. J., Physica 25 (1959) 645.

6) Yamamoto, T., Phys. Rev. 119 (1960) 701. 
7) Mazur, P., Physica 43 (1969) 533.

8) Kato, T., Perturbation Theory for Linear Operators, Springer-Verlag (New York, 1966).

9) Caspers, W.J., Van de Braak, H.P., Verbeek, P.W. and Verstelle, J.C., Physica 53 (1971) 210.

10) Valkering, T.P. and Caspers, W.J., Physica 63 (1973) 113.

11) Valkering, T.P., thesis, Twente University of Technology, Enschede (1973).

12) Bongaarts, P.J. M. and Siskens, Th. J., Physica 71 (1974) 529.

13) Valkering, T.P., Physica 53 (1971) 117. 